\title{
Evaluation of outpatient experience with vitreoretinal surgery
}

\author{
Cynthia S Cannon, Jeffrey G Gross, Ian Abramson, William J Mazzei, William R Freeman
}

\begin{abstract}
The experience of 55 consecutive individuals undergoing outpatient vitreoretinal surgery was evaluated. Objective variables, including preoperative and intraoperative information, subjective postoperative pain, and discomfort were measured with a previously validated $100 \mathrm{~mm}$ visual analogue scale. Patients also ranked the overall experience. Average pain and discomfort scores in the recovery room were 21.8 and 22.6 and overnight were 26.7 and 30.4 (scale 0 to 100), respectively. Eighty eight per cent of subjects were satisfied with the experience. Elevated pain and discomfort scores were statistically correlated with scleral buckling, prolonged surgical or recovery room time, requirement for parenteral pain medications, and high intraocular pressure on the first postoperative visit. None of the patients needed further hospital treatment. This study suggests that vitreoretinal surgery in an appropriately selected population does not require routine inpatient care.
\end{abstract}

Health care has become increasingly focused on cost reduction, particularly on reduction of inpatient care, and conversion of traditionally inpatient procedures, such as cardiac catheterisation,' into outpatient treatment. However, hospital care remains common for patients undergoing vitreoretinal surgery for the repair of retinal detachment and other diseases of the retina. Isernhagen and associates ${ }^{2}$ recently reported on a series of 200 consecutive inpatients undergoing vitreoretinal surgery. They concluded that many factors, including excessive pain, nausea, and vomiting, occurred frequently enough to necessitate routine overnight stay in hospital.

Outpatient procedures may reduce costs significantly and may offer psychological benefit to the patient. Recent experience at our institution has suggested that, with the exception of small children and diabetics, many patients tolerate outpatient vitreoretinal surgery quite well if proper precautions are taken to prevent complications which require hospital care. It was our impression that most of the cited reasons for impatient care, such as severe nausea, are often the result of systemic anaesthetics rather than the surgery itself. We prefer local anaesthesia in conjunction with mild, short acting intravenous sedation for vitreoretinal surgery. General anaes- thesia, though conducive to patient immobility during long procedures, may require a longer, more uncomfortable postoperative recovery period. ${ }^{3}$ To maintain adequate anaesthesia during the longer operations we found that irrigation of the peribulbar tissue with a $4 \%$ lignocaine with $0.75 \%$ bupivacaine solution through a blunt cannula was efficacious. Increased intraocular pressure can usually be controlled with perioperative carbonic anhydrase inhibitors.

Scleral buckling is another significant source of pain. Unlike the retina, which is devoid of pain fibres, the sclera is innervated by free nerve endings of the trigeminal nerve. ${ }^{4}$ In many of our patients undergoing vitreoretinal surgery we no longer perform extensive scleral buckling. Patients referred for proliferative vitreoretinopathy at our institution usually have already received encircling scleral elements which we rarely revise. In eyes requiring vitreous surgery and encircling buckles, a relatively small 7.5-9.0 $\mathrm{mm}$ encircling element is inserted to support the vitreous base and relieve any residual traction internally. We usually treat uncomplicated retinal detachments by segmental buckling performed under local anaesthesia.

We undertook a prospective study to analyse patient discomfort and experience with outpatient vitreoretinal surgery using objective and subjective data obtained from consecutive outpatients. We sought to determine (1) what percentage of patients tolerate outpatient vitreoretinal surgery, (2) the factors that are associated with a favourable or unfavourable outpatient experience, and (3) overall patient satisfaction with the outpatient setting.

\section{Materials and methods}

Subjects included 55 consecutive patients undergoing vitreoretinal surgery by a single surgeon (WRF). Patients had the choice of general or local anaesthesia with intravenous sedation using primarily fentanyl citrate and midazolam hydrochloride. Patients were encouraged to have local anaesthesia to reduce postoperative discomfort and hasten recovery. Before discharge, the intraocular pressure in those patients who received intraocular gas was measured and found to be below $35 \mathrm{~mm} \mathrm{Hg}$. All patients had received intravenous or oral acetazolamide immediately postoperatively.

Two groups of patients were studied. One 
Table 1

Preference statements; please choose one.

(1) I was comfortable going home on the same day as my surgery knowing I would see the doctor today. There was no reason for $m$ to stay overnight in the hospital

(2) I was concerned about leaving the hospital on the same day as my surgery. I would have felt more comfortable if I had stayed overnight but it worked out okay.

(3) I should have stayed overnight at the hospital after my surgery. I was in considerable pain and discomfort at home.

consecutive series of patients were retrospectively contacted by letter. Another consecutive series of patients were studied prospectively, interviewed in the clinic on the first postoperative day with medical data collected at that time. Patients under 15 years old were excluded from the study.

The preoperative objective information included age, sex, number of previous surgeries on the operated eye, and medical history. The intraoperative information included type of anaesthesia (general or local), type of procedure performed (trans par plana vitrectomy, scleral buckling, intraocular long-acting gas injection and/or intraocular silicone oil injection), operated eye, and surgical time in minutes. In all cases, gas or silicone oil injections were performed in conjunction with pars plana vitrectomy surgery. No patients in this study underwent pneumatic retinopexy as that procedure is performed in the clinic at our institution. The postoperative factors analysed were time from completion of operation to hospital discharge in minutes, type of analgesia received in the recovery room, ocular status on the first postoperative day as assessed by the surgeon, including intraocular pressure in the operated eye, status of the cornea, degree of inflammation, attachment of retina, and presence of vitreous haemorrhage.

Data obtained after surgery included type and amount of analgesics taken, whether the ophthalmologist was called after discharge from hospital, a three-point preference scale regarding outpatient treatment (Table 1) and a series of standardised visual analogue scales to measure pain and discomfort (Fig 1) for the periods in the recovery room, overnight and, in the case of prospective patients, at the time of interview.

Predictors, defined as preoperative, intraoperative, or postoperative, were compared individually with each of the subjective outcome measures using $\chi^{2}, t$ test, or simple regression, as appropriate. All data were analysed using StatView 512 (BrainPower, Inc, Calabasas, CA, USA) on a Macintosh SE computer.

Results

A total of 55 patients, 33 males and 22 females, ages 22 to 90 , were studied - 37 patients, 22

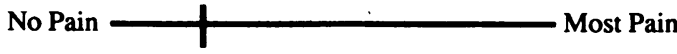

Figure 1 Example of the highly validated visual analogue scale for measuring pain. The patient is asked to place a vertical mark across the horizontal line indicating intensity of pain with 'most pain' indicating the most severe pain the patient has experienced. The scale is linear with zero assigned to 'no pain' and 100 assigned to 'most pain'. In this case, the score is 20
Table 2 Medical conditions and procedures performed

\begin{tabular}{lrr}
\hline & Total number & Percent \\
\hline Medical conditions & & \\
None & 31 & $56 \cdot 0$ \\
Diabetes & 5 & $9 \cdot 1$ \\
Hypertension & 14 & $25 \cdot 0$ \\
Heart disease & 6 & $10 \cdot 9$ \\
Acquired immunodeficiency syndrome & 4 & $7 \cdot 3$ \\
Procedures & 48 & $87 \cdot 3$ \\
Trans par plana vitrectomy & 23 & $41 \cdot 8$ \\
Scleral buckle & 17 & $31 \cdot 0$ \\
Intraocular gas & 17 & 31.0 \\
Silicone oil & & \\
\hline
\end{tabular}

Table 3 Ocular status on postoperative day one

\begin{tabular}{lcc}
\hline & Number of patients & Percent \\
\hline Intraocular pressure $>40 \mathrm{~mm} \mathrm{Hg}$ & 2 & $3 \cdot 6$ \\
Oedematous cornea & 19 & $36 \cdot 0$ \\
Retina attached & 52 & 98.0 \\
Vitreous haemorrhage & 7 & $13 \cdot 0$ \\
Inflammation & 7 & $13 \cdot 2$ \\
$\quad$ None & 21 & $39 \cdot 6$ \\
Mild & 18 & $34 \cdot 0$ \\
Moderate & 7 & $13 \cdot 2$ \\
Severe & & \\
\hline
\end{tabular}

Table 4 Pain and discomfort score summary

\begin{tabular}{llllll}
\hline & Mean & $S D$ & Range & No. $>75$ & $\%>75^{\star}$ \\
\hline Recovery room & & & & & \\
Pain & $21 \cdot 8$ & $29 \cdot 6$ & $0-100$ & 6 & $11 \cdot 0$ \\
Discomfort & 22.6 & 30.3 & $0-100$ & 7 & $12 \cdot 7$ \\
Overnight & 26.7 & $30 \cdot 7$ & $0-98$ & 7 & 12.7 \\
$\quad$ Pain & $30 \cdot 4$ & $29 \cdot 7$ & $0-99$ & 7 & 12.7 \\
Discomfort & 30.4 & &
\end{tabular}

^Indicates percentage of patients with a pain or discomfort score over 75 which indicates moderately intense pain or discomfort.

males and 15 females, were studied prospectively. Their average age was 54 years, range 22 to 86. Eleven males and seven females responded to the mailed questionnaire. Five males and two females did not respond, were unreachable, refused the study, or did not fill out the questionnaire correctly. The average age of these patients was 62.5 years old (ranging from 24 to 90 ). All but two participating patients had local retrobulbar anaesthesia and two thirds had a history of previous eye surgery. Underlying medical problems and procedures and their frequencies are listed in Table 2 . Surgical time ranged from 30 to 245 minutes, and averaged 120 minutes.

Time to hospital discharge averaged 131 minutes, ranging from 45 to 375 minutes. Nine per cent of patients required parenteral analgesia in the recovery room. Ocular status on the first postoperative day is summarised in Table 3.

Only one patient had pain that required parenteral analgesia after hospital discharge. Ten per cent of the patients called the physician between discharge and the first postoperative day visit. Seventy eight per cent of patients chose statement number 1 that indicated they preferred outpatient treatment to the option of an overnight stay in hospital, with an additional 10 per cent choosing statement number 2 . Pain and discomfort scores from the visual analogue scales are summarised in Table 4. A score of more than 75 was taken to indicate severe pain. ${ }^{56}$ No patients required readmission to hospital following their initial discharge.

Several objective measures significantly correlated with elevated pain and discomfort scores: scleral buckling $(p=0.02)$, prolonged 
surgical time $(p=0.03)$, prolonged recovery room time $(p=0 \cdot 04)$, requirement of parenteral analgesia in the recovery room $(p=0.005)$, post-discharge telephone call to the physician $(p=0.02)$, and elevated intraocular pressure as measured on the first postoperative day $(p=$ $0.004)$. The retrospective data were compared with the prospective data and no difference in conclusions existed. Intraocular pressure elevation did not cause visual loss in any patient.

\section{Comment}

The results of this study indicate that vitreoretinal surgery can be performed as an ambulatory procedure in the majority of our patient population. Our study population of outpatients was preselected to include individuals deemed by the surgeon to be good outpatient risks. We excluded poorly controlled insulin-dependent diabetics who are known to have difficult postoperative courses regardless of the type of operation, particularly those undergoing general anaesthesia. ${ }^{7}$ They are routinely treated as inpatients at our institution not because of postoperative pain and discomfort but rather to optimise postoperative medical management of their underlying disease. We studied both a consecutive retrospective and prospective series of patients; analysis of both groups and the total population gave the same results.

This study suggests that routine inpatient care of all vitreoretinal surgery patients is unnecessary using newer anaesthetic techniques. ${ }^{8}$ A significant proportion can undergo surgery in the outpatient setting, and these patients appreciate such a treatment.

Our results contrast with those of Isernhagen ${ }^{2}$ who studied a strictly inpatient population and concluded that an overnight stay in hospital was required in nearly all vitreoretinal surgical patients because of postoperative pain and discomfort. Our study population differs only in the proportion of diabetics $(9 \cdot 1 \%$ in our study versus $35 \%$ in theirs), because complicated, insulindependent diabetics are routinely managed postoperatively as inpatients at our institution.

We attempted a more rigorous design to measure the subjective pain and discomfort of patients through the use of the visual analogue scale, a linear scale used by patients to indicate visually their level of pain or discomfort, and a choice of preference statements. However, the visual analogue scale, by translating a continuous-scale perception into a continuous scale on paper, is considered the most valid and reliable method for this purpose. ${ }^{5}$ This tool has been successfully used and validated in studies regarding pain associated with dental work, ${ }^{69}$ rheumatoid arthritis, ${ }^{10}$ and elective gallbladder surgery. "We were careful to ensure that all patients studied had adequate vision in at least one eye and adequate understanding of the scale to ensure reliability.

Certain variables are correlated with higher pain and discomfort during the immediate postoperative 24 hours. Each deserves comment: (1) scleral buckling; this would be expected to induce more pain and discomfort due to intraoperative traction of the extraocular muscles, and the presence of a foreign body on the sclera. (2) Longer surgical time; this is in agreement with another study ${ }^{12}$ that cited factors associated with unplanned admissions following outpatient ophthalmic procedures. (3) Longer time to discharge; reasons why the patient stayed longer in the recovery room were not easily accounted for. However our postoperative questioning of patients suggests that they may be related to perioperative pain and discomfort, problems in ambulation, non-medical problems (eg transportation), and other factors. (4) Those who required parenteral analgesia in the recovery room were experiencing pain that could not be relieved by oral medications and their scores, not surprisingly, reflected this observation. (5) Reasons for a late night call to the doctor did not result in readmission to hospital; the calls involved reassurance, postoperative advice, and instructions on the use of medications. (6) High intraocular pressure $(>40 \mathrm{~mm} \mathrm{Hg})$ is known to be painful and patients with increased intraocular pressure on the first postoperative day would be expected to have higher intraocular pressure immediately postoperatively ${ }^{13}$ which contributed to their pain. It is of note that elevated intraocular pressure did not cause vision loss in any of our patients.

Though the above significant correlations were found they do not necessarily predict a need for inpatient care, but rather provided some indicators of an increased need for analgesia or personal follow up. Our postoperative questioning suggested that the outpatient setting was associated with greater vigilance in maintainence of special positioning. This was secondary to the presence of supportive family members compared with the alternative of busy hospital nurses who may be less informed about the importance of positioning. It is possible that this would result in improved surgical outcome.

It is interesting to note that there was no significant difference in pain or discomfort scores between genders or ages, nor between those who did and did not receive silicone oil intraocularly.

Our study agrees with Wilson and Barr ${ }^{14}$ who retrospectively reviewed a series of vitreoretinal surgery cases performed under general anaesthesia and in hospital with local anaesthesia and outpatient treatment. Our results apply to patients who meet our criteria for being good outpatient risks, including the absence of serious or uncontrolled concomitant medical conditions and the ability to tolerate and cooperate with local anaesthesia during a potentially long procedure. We prefer smaller encircling elements and emphasise internal relief of traction over extensive scleral buckling. In many cases, our patient population entailed re-operations or other surgery where scleral buckling was not employed.

Patients appropriate for outpatient management must be able to follow postoperative directions regarding special positioning, medications, follow up, and other relevant instructions. They must have a place to stay, a responsible party to provide transport, and a postoperative carer in close proximity for at least the first 24 postoperative hours. In our experience, insulin- 
dependent diabetics are generally not good candidates for ambulatory vitreoretinal surgery. Paediatric patients without serious underlying medical problems can tolerate outpatient surgery but the need for general anaesthesia generally necessitates a longer recovery room stay.

Dr Gross is presently at the University of South Carolina School of Medicine, Columbia, SC, USA.

This paper was presented at the Association for Research in Vision and Ophthalmology, Sarasota, FL, 29 April-4 May 1990.

This work was supported in part by NIH Grant EY07366,

rant from the University of California, the Jeanette Rubin Family grant from the University of California, the Jeanette Rubi

1 Block PC, Ockene I, Goldberg RJ, et al. A prospective randomized trial of outpatient versus inpatient cardiac catheterization. $N$ Englf Med 1988; 319: 1251-5.

2 Isernhagen RD, Michels RG, Glaser BM, de Bustros S, Enger C. Hospitalization requirements after vitreoretinal surgery. Arch Ophthalmol 1988; 106: 767-70.

3 Mecca RS. Postanesthesia recovery. In: Barash PG, Cullen BF, Stoelting RK, eds. Clinical anesthesia. Philadelphia: JP Lippincott, 1989: 1419-25.

4 Miller NR. Anatomy and physiology of the trigeminal nerve. In: Miller NR ed Walsh and Hoyt's clinical neuroophthalmology. 4th ed. Baltimore: Williams \& Wilkins, 1985: 1008-9.

5 Ohnhaus EE, Adler R. Methodological problems in the measurement of pain: a comparison between the verbal rating scale and the visual analogue scale. Pain 1975; 1: 379 84.

6 Beaver WT, Forbes JA, Schackleford RW A method for the 12-hour evaluation of analgesic efficacy in outpatients with post-operative oral surgery pain. Pharmacotherapy 1983; 3 : post-operati

7 Karhunen U, Summanen P, Laatikainen L. Concomitant problems to the anaesthesia of diabetic vitrectomy patients. Acta Ophthalmol 1987; 65: 190-6.

8 Mein CE, Woodcock MG. Local anesthesia for vitreoretinal surgery. Retina 1990; 10: 47-9.

9 Seymour RA, Meechan JG, Blair GS. An investigation into post-operative pain after third molar surgery under local analgesia. Brf Oral Maxillofac Surg 1985; 23: 410-8.

10 Callahan LF, Brooks RH, Summary JA, Pincus T. Quantitative pain assessment for routine care of rheumatoid arthritis patients, using a pain scale based on activities of daily living and a visual analog scale. Arthritis Rheum 1987; 30: $630-6$.

11 Taenzer P, Melzack R, Jeans ME. Influence of psychological factors on post-operative pain, mood and analgesic require-

12 Freeman LN, Schachat AP, Manolio TA, Enger C. Multivariate analysis of factors associated with unplanned admission in 'outpatient' ophthalmic surgery. Ophthalmic Surg 1988; 19: 719-23.

13 Gross JG, Meyer DR, Robin AL, Filar AA, Kelley JS. Increased intraocular pressure in the immediate postopera-
tive period after extracapsular cataract extraction. $A m \mathcal{F}$ Ophthalmol 1988; 105: 466-9.

14 Wilson D, Barr CC. Outpatient and abbreviated hospitalization for vitreoretinal surgery. Ophthalmic Surg 1990; 21: 119-22. 